\title{
Atom Probe Tomography of Engineered Nanostructures with Complex Field Evaporation Behavior
}

\author{
K.T. Henry,* A.A. Herzing* and I.M. Anderson* \\ * Surface and Microanalysis Science Division, National Institute of Standards and Technology, \\ 100 Bureau Dr., Gaithersburg, MD 20899-8371, karen.henry@nist.gov
}

Atom probe tomography (APT) is a powerful technique for three dimensional (3D) chemical analysis with subnanometer resolution [1], and has provided unique insights into phase transformations, grain boundary segregation, and interfacial reactions. Recent advancements in APT instrumentation have extended the technique to lower conductivity materials such as semiconductors and organics. As device structures continue to decrease in size and to take on complex non-planar architectures, 3D characterization at the atomic level is necessary to improve nanoelectronic technology performance. APT provides the required spatial resolution and chemical sensitivity to characterize these next-generation devices; however, these complex structures, which feature a juxtaposition of disparate classes of material, pose a challenge for the technique. Metal, semiconducting, and dielectric materials with layer thicknesses as little as a nanometer can be present in such device structures, and corresponding variations in evaporation field lead to a nonhemispherical specimen endform. Small variations in radius of curvature at the specimen apex can distort the positions of the atoms in the reconstructed volume, thus understanding how the surface topology evolves is prerequisite to accurate reconstruction of these complex nanoscale structures. The evolution of the surface topology can be analyzed by interrupting data acquisition at various stages of the APT experiment and characterizing the specimen endform via electron tomography. Transmission electron microscopy (TEM) thereby provides a complementary technique to improve the accuracy of APT reconstructions of these complex devices [2].

The challenges for APT characterization of next-generation nanoelectronic devices can be illustrated by analysis of a model dielectric/metal thin film stack on a silicon substrate. For this study, APT was used to quantify subtle compositional fluctuations at or near grain boundaries in order to better understand the polymorphic A1-to- $L 1_{0}$ phase transformation in FePt thin films. This simple phase transformation is of significant interest in the area of small-bit-size media for the development of a material to overcome the superparamagnetic limit [3]. $L 1_{0}$-ordered FePt is a leading material for thermally stable, small-bit magnetic storage media because this phase has a high uniaxial magnetocrystalline anisotropy $K_{u}$ [4]. However, when FePt is sputter deposited, the thin film typically adopts the high temperature, soft-magnetic disordered face-center cubic A1 structure. Subsequent annealing is required to transform the material into the desired magnetically hard $L 1_{0^{-}}$ ordered phase. Modeling predictions have suggested preferential segregation at the nanometer length scale occurs during this polymorphic transformation [5].

A series of FePt thin films were sputter-deposited between silicon nitride layers and annealed at various times and temperatures to facilitate the A1-to- $L 1_{0}$ phase transformation. During conventional annealing of $\mathrm{FePt}$ in the temperature range of $500{ }^{\circ} \mathrm{C}$ to $800{ }^{\circ} \mathrm{C}$, grain coarsening occurs and the narrow grain size distribution required for ultra-high density storage applications is destroyed [6]. To mitigate grain growth during the phase transformation, plasma arc pulses of millisecond-scale duration are used to thermally anneal the FePt thin films. However, a degradation of order parameter 
and coercivity is observed for films pulsed as few as five times. APT was used to characterize the annealed $\mathrm{Si} / \mathrm{FePt}$ interface. The reduction in the two properties of the film has been attributed to the interdiffusion at the film-substrate interface and the formation of iron oxide clusters at the FePt grain boundaries, as shown in Figure 1. The gray isoconcentration surface illustrates the boundary between the silicon substrate and FePt thin film. A proximity histogram indicates the diffusion of Si into the film. A significant concentration of oxygen is also observed at the interface. Iron oxide clusters are highlighted by a yellow isoconcentration surface and are arranged near grain boundaries.

Of significance to APT analysis of next-generation nanoelectronics, the metal-nitride interfaces posed a challenge for APT data acquisition because of their disparate evaporation fields. Attempts to analyze the film stack in plan-view resulted in specimen fracture, and the data could be acquired only with the specimen oriented in the plane of the film such that the layers evaporated concomitantly.

[1] M.K. Miller et al., Atom Probe Field Ion Microscopy, Clarendon Press, Oxford 1996.

[2] B.P. Gorman, Microsc. Microanal. 13 (Suppl. 2) (2007) 1616.

[3] D. Weller et al., IEEE Trans. Magn. 36 (2000) 10.

[4] B. Zhang and W.A. Soffa, Scripta Metall. 30 (1994) 683.

[5] C. Srivastava et al., J. Appl. Phys. 102 (2007) 104310-1.

[6] H. Uwazumi et al., IEEE Trans. Magn. 37 (2001) 1595.

[7] This research was partially supported by the National Science Foundation under contract NSFDMR-0529369. The aid of Dr. Greg Thompson at the University of Alabama is gratefully acknowledged.

(a)

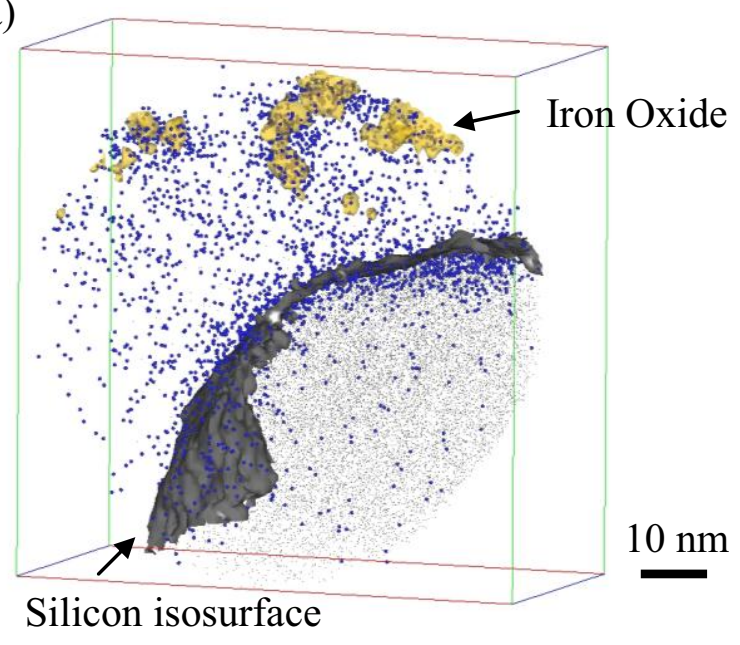

(b)

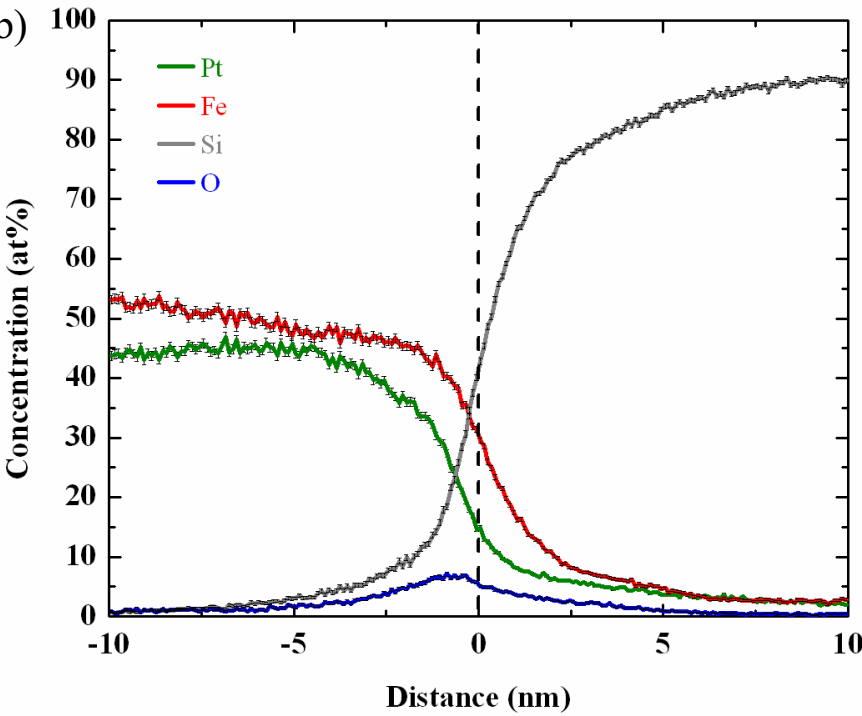

FIG. 1. APT reconstruction of phase and solute distributions at the substrate-film interface for a 100 -nm-thick film of FePt deposited onto silicon and annealed with three plasma-arc pulses of 100 ms duration. (a) APT atom map augmented with isoconcentration surfaces. The gray points are $\mathrm{Si}$ atoms. The blue spheres are $\mathrm{O}$ atoms. The iron oxide isoconcentration surfaces surround regions with concentrations greater than 3 at.\%. (b) A proximity histogram showing interdiffusion and oxygen segregation at the film-substrate interface. The location of the silicon isoconcentration surface in (a) is indicated by the vertical dashed line. 